\title{
Dislocation Rates following Anterior Approach THA: The Role of Functional Pelvic Tilt
}

\author{
Preetesh Patel, MD ${ }^{1}$ Colin McNamara, MD, MBA ${ }^{1}$ Eric Slotkin, MD ${ }^{2}$ Amar Mutnal, MD ${ }^{1}$ \\ Wael Barsoum, MD ${ }^{1}$ Juan C. Suarez, MD ${ }^{1}$
}

${ }^{1}$ Department of Orthopaedic Surgery, Cleveland Clinic Florida,
Weston, Florida
2 Orthopaedic Associates of Reading, Ltd., Reading, Pennsylvania

Address for correspondence Preetesh Patel, MD, Department of Orthopaedic Surgery, Cleveland Clinic Florida, 2950 Cleveland Clinic Boulevard, Weston, FL 33331 (e-mail: patelp4@ccf.org).

J Hip Surg 2017;1:194-199.

\begin{abstract}
Keywords

- accuracy

- complication

- component position

- fluoroscopy

- hip arthroplasty

Various target zones for acetabular cup placement have been suggested to minimize dislocation following total hip arthroplasty (THA), though dislocations occur despite proper positioning. The authors have reported accuracy of fluoroscopic guidance in cup positioning during direct anterior approach (DAA) THA when using a standardized fluoroscopic technique functional pelvic tilt. They believe that cup placement with regard to functional pelvic tilt, rather than static reference frames, will offer improved stability. A cohort of 1,597 fluoroscopy-assisted DAA primary unilateral THA patients was prospectively followed for a minimum of 1 year and component position measured radiographically. Target cup position was based off the standing anteroposterior pelvis while using conventional safe zone parameters. Dislocation rate was assessed. Average follow-up was 13.1 months (range 1-6 years). The mean abduction angle was $37.7^{\circ}$ and the mean anteversion angle was $16.2^{\circ}$. Overall, 1,517 (95.0\%) fell within the targeted abduction range, 1,528 (95.7\%) fell within the targeted anteversion range, and 1,456 (91.2\%) simultaneously met both criteria. There were nine dislocations, eight within the combined safe zone, for a dislocation rate of $0.56 \%$. Fluoroscopy can improve accuracy and precision of cup placement in DAA THA. Conventional safe zone parameters applied using functional pelvic tilt resulted in a low dislocation rate, with most dislocations occurring within the safe zone. Using a dynamic functional safe zone may further reduce dislocation rates after DAA THA, though other factors that contribute to instability must be accounted for in future studies. This study had a III level of evidence.
\end{abstract}

Dislocation following total hip arthroplasty (THA) is a leading cause for early revision, with significant clinical and economic impact. ${ }^{1}$ Several factors contribute to dislocation that include, but are not limited to, surgical approach, soft tissue balancing, patient anatomy, and component positioning. ${ }^{2-7}$ Considerable effort has been made to identify a safe zone for acetabular component position that would minimize risk of dislocation after primary THA. ${ }^{8}$ The commonly referenced Lewinnek safe zone suggests cup abduction and anteversion

received

August 23, 2017

accepted after revision

January 19, 2018

published online

March 19, 2018 of $40^{\circ} \pm 10^{\circ}$ and $15^{\circ} \pm 10^{\circ}$, respectively. ${ }^{9}$ However, cups within this and other described safe zones are not immune to dislocation. Some argue that a true safe zone may not exist. ${ }^{10-12}$ Particular attention must be placed on the reference planes for cup measurement by which a safe zone is derived. ${ }^{13}$ The Lewinnek safe zone is based on cup measurements using Murray's radiographic definition of acetabular orientation referenced off the anterior pelvic plane (APP), as opposed to the functional (coronal) plane, which is based on
Copyright $\odot 2017$ by Thieme Medical Publishers, Inc., 333 Seventh Avenue, New York, NY 10001, USA. Tel: +1(212) 584-4662.
DOI https://doi.org/ $10.1055 / \mathrm{s}-0038-1635101$. ISSN 2472-8446. 
the longitudinal axis of the body. ${ }^{14}$ Pelvic orientation is specific to each patient and dynamically changes with patient position. ${ }^{15}$ Failure to consider these relationships of the pelvis to the longitudinal axis of the body may lead to improper functional orientation of the acetabular cup and explain why some dislocations occur despite acetabular components believed to be within the Lewinnek safe zone. $^{16,17}$

The longitudinal axis of the body can be measured in the supine, seated, and standing positions. In each of these positions, there are changes in pelvic tilt, which is defined as the angle between the line connecting the midpoint of the sacral plate to the axis of the femoral heads and the vertical axis. ${ }^{15}$ Pelvic tilt can vary among patients, between sexes, and during different activities of daily living and sportspecific activities. ${ }^{18}$ In most patients, going from supine to standing causes a small increase in anteversion of the acetabular component due to relative increase in posterior pelvic tilt. During direct anterior approach (DAA) THA, intraoperative fluoroscopy is typically obtained with the patient supine on the operating table with reference to the functional plane (assuming a level operative table and orthogonal fluoroscopic image), essentially recreating supine anteroposterior (AP) pelvis radiograph; however, this may not represent the best functional position of the pelvis and the acetabulum. ${ }^{19}$ It is possible that standing radiographs may better integrate the dynamic influences of periarticular musculature and sagittal balance. ${ }^{20}$ Patients with ankylosing spondylitis or other diseases that drastically affect sagittal balance ( $>20^{\circ}$ from neutral) can be at higher risk of dislocation due to cup malpositioning when referencing the static APP. ${ }^{21}$

It is our practice to obtain preoperative standing AP pelvis and hip radiographs. Intraoperative fluoroscopy is utilized to replicate the pelvic orientation of the preoperative standing film, which provides the basis for cup placement. This process inherently accounts for patient-specific variations in pelvic tilt without the need for computer navigation, advanced imaging, lateral pelvic radiographs, or nomograms. The purpose of this study was to evaluate whether cup placement with regard to functional pelvic tilt during DAA THA using a standardized fluoroscopic technique will result in improved postoperative stability. As there is no universally accepted pelvic reference frame in which to base acetabular component position, an acetabular safe zone based on the standing functional plane may more accurately predict stability after primary DAA THA.

\section{Material and Methods}

After obtaining institutional review board approval and using our prospectively constructed database, we identified 1,597 consecutive AA THA surgeries performed via a DAA by two fellowship-trained surgeons from March 1, 2010 through March 1, 2016. Average follow-up was 13.1 months (range 1-6 years). Both surgeons (P.P. and J.C.S.) were 3 years removed from fellowship with no formal training in DAA during residency or fellowship at the start of collection. The database contained date of operation, performing surgeon, laterality of the operation, most recent follow-up, cup anteversion and abduction angles, body mass index, and the postoperative complication of dislocation. Intraoperative pelvic position was determined by comparing the fluoroscopic image and the standardized preoperative standing AP pelvis radiograph. The $C$-arm was angled cranially or caudally and the beam properly centered to reproduce the pelvic orientation from the preoperative radiograph, using the obturator foramen ratio and coccyx-to-symphysis distance as references. This allowed us to account for positional changes in pelvic tilt with patient supine, which could result in suboptimal cup positioning when changes in tilt are extreme ( $\mathbf{- F i g . 1}$ ). Neutral pelvis rotation was achieved by proper patient positioning on the operative table with minor adjustments made with the C-arm so that the coccyx was aligned with the pubic symphysis and the obturator foramina were symmetric. Intraoperative cup placement was gauged under the fluoroscopic AP pelvis view with a target orientation of $40^{\circ} \pm 10^{\circ}$ abduction and $15^{\circ} \pm 10^{\circ}$ anteversion. All measurements of acetabular component positioning were obtained from a standardized standing digital AP pelvis radiograph taken at least 2 weeks postoperatively. Patients were assessed for postoperative dislocation through clinic follow-up and/or phone contact.

\section{Acetabular Component Measurement}

Impax (version 6.0, AGFA Health Care) imaging software was used to calculate acetabular component inclination and version angles using methodology previously described. ${ }^{22}$ Radiographic acetabular version angle was calculated from the arcsine of the ratio of the minor and minor axes of the ellipse created from the rim of the cup. Inclination was determined by measuring the angle of the major axis of the ellipse and the interteardrop line.

\section{Results}

For the 1,597 DAA THAs, average inclination and anteversion measured $37.7^{\circ} \pm 4.8^{\circ}$ and $16.2^{\circ}{ }^{\circ} \pm 5.4^{\circ}$, respectively (-Fig. 2). Overall, 1,517 (95.0\%) fell within the targeted abduction range, 1,528 (95.7\%) fell within the targeted anteversion range, and 1,456 (91.2\%) simultaneously met both criteria. Average follow-up was 13.1 months. There were nine dislocations for a dislocation rate of $0.56 \%$ (-Table 1). Eight were within the combined safe zone. Eight occurred within the first 8 weeks and four ultimately required revision for persistent instability.

\section{Discussion}

Our dislocation rate of $0.56 \%$ compares favorably to dislocation rates previously reported regardless of approach. ${ }^{23,24}$ Cases within the learning period were also included. Dislocation rates range between $0.6 \%$ to $1.0 \%$ for DAA and $0.3 \%$ to $0.6 \%$ for direct lateral approach. ${ }^{5,25}$ Posterior approach has generally been associated with higher dislocation rates of $1.7 \%$ to $5.3 \%$, but recent literature has demonstrated 

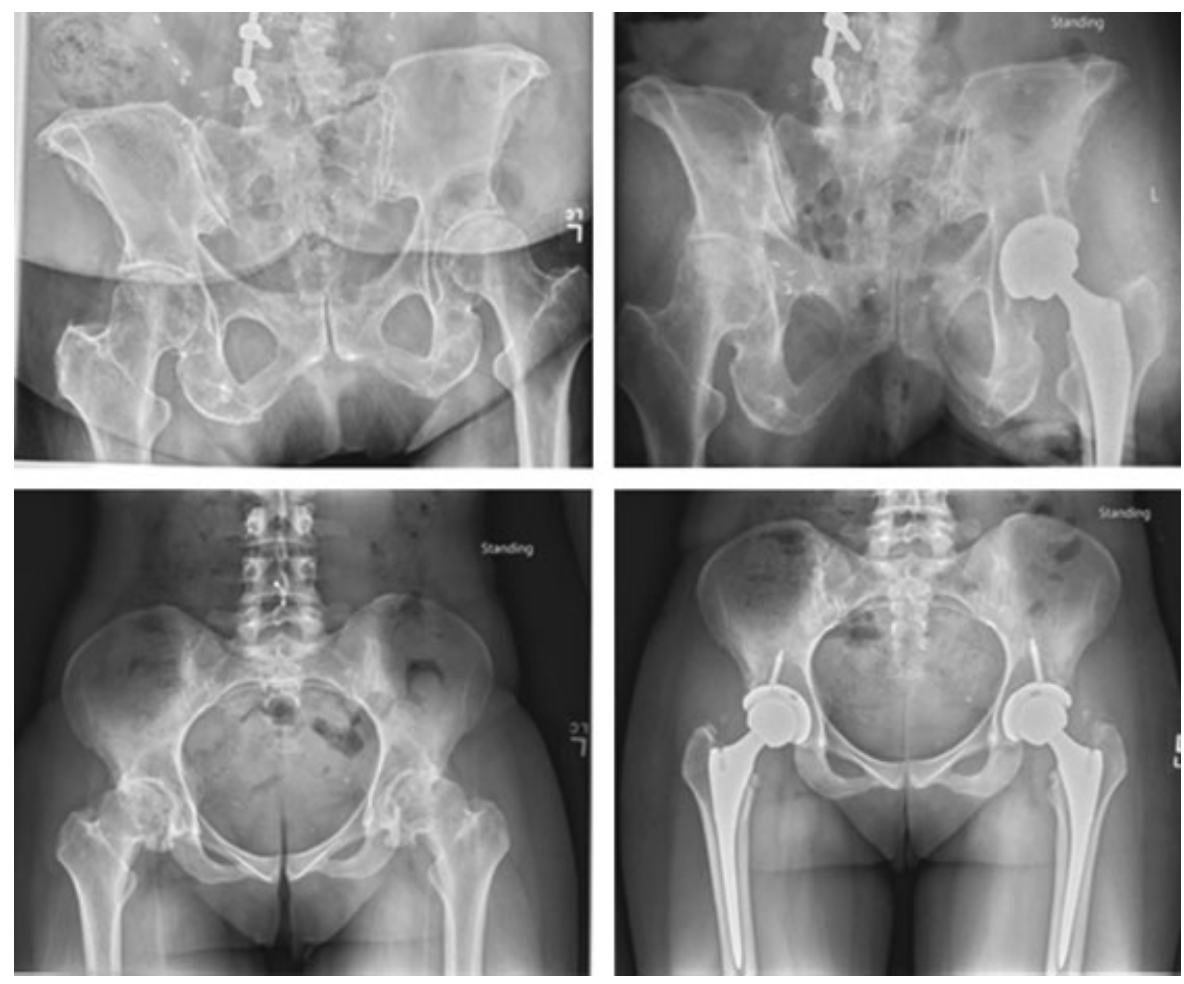

Fig. 1 Examples of extreme posterior pelvic tilt and anterior pelvic tilt with cups placed on the defined safe zone within the corresponding pelvic tilt.

improved stability with posterior capsular repairs. ${ }^{26,27}$ Another study reported dislocation rates of $0.55 \%, 2.18 \%$, and $3.23 \%$ for direct lateral, anterolateral, and posterior approaches, respectively. ${ }^{28}$ We previously found that our cup position accuracy improved yearly; ${ }^{22}$ however, we did not analyze dislocation rates or cup orientation parameters with regard to surgeon experience in this study. We believe that fluoroscopy is inexpensive and invaluable tool to improve accuracy and precision of cup placement in DAA
THA and that the functional pelvic plane possibly provides a better framework from which to formulate an acetabular component safe zone.

Intraoperative navigation aids in component positioning, yet still has limitations. Navigation systems reference anatomic landmarks to define the APP; however, they do not incorporate preoperative pelvic tilt. ${ }^{16}$ The APP itself is a static measurement. Lembeck et al ${ }^{17}$ demonstrated that pelvic tilt significantly affected the accuracy of navigation systems due

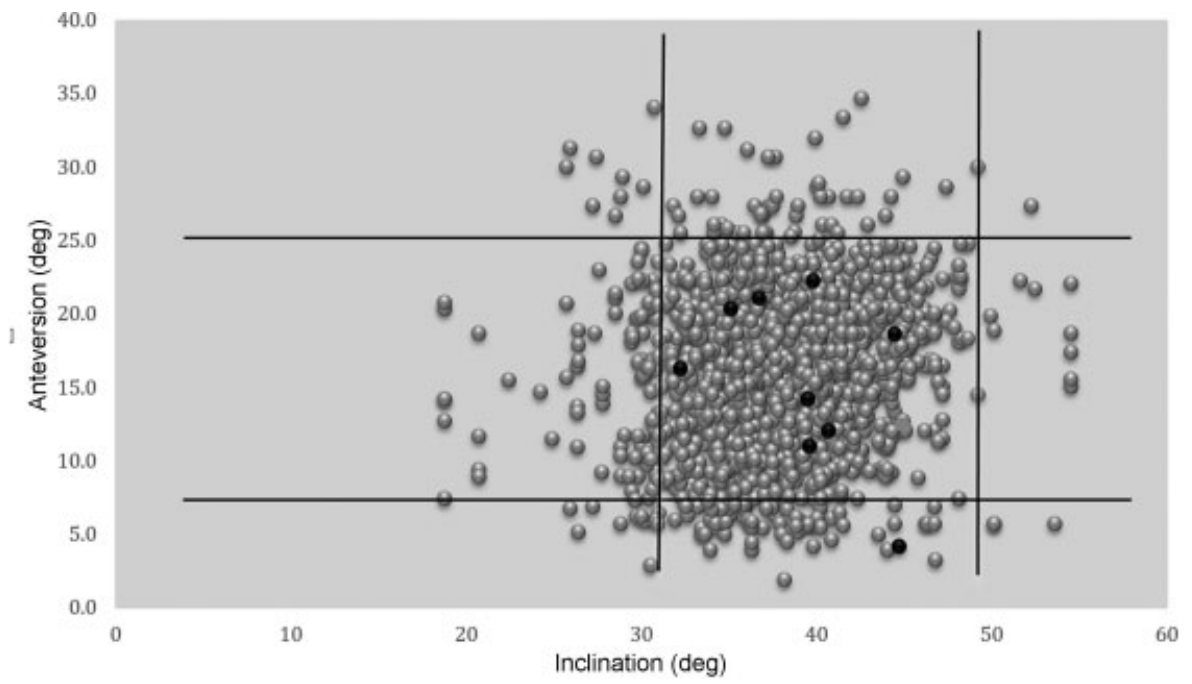

Fig. 2 Scatterplot demonstrating the number of direct anterior approach total hip arthroplasties within defined target ranges, indicated by the black lines, for both inclination $\left(30^{\circ}-50^{\circ}\right)$ and anteversion $\left(5^{\circ}-25^{\circ}\right)$. Dislocations are represented in black. 
Table 1 Dislocations demonstrating the time of dislocation and if a subsequent revision was done

\begin{tabular}{|c|c|c|c|c|c|c|}
\hline Patient & Inclination & Version & $\begin{array}{l}\text { Number of } \\
\text { dislocations }\end{array}$ & Time to dislocation & Revised? & Notes \\
\hline 1 & 44.7 & 22.3 & 3 & $2 \mathrm{~m} ; 3 \mathrm{~m} ; 4 \mathrm{~m}$ & Yes & $\begin{array}{l}2 x \text { spontaneous reduction; } \\
1 \times \text { closed reduction }\end{array}$ \\
\hline 2 & 32.2 & 12.1 & 2 & $4 w ; 6 w$ & Yes & $2 x$ closed reduction \\
\hline 3 & 44.4 & 20.4 & 2 & $25 \mathrm{~m} ; 25.5 \mathrm{~m}$ & Yes & $2 x$ closed reduction \\
\hline 4 & 39.5 & 21.1 & 1 & $2 \mathrm{~m}$ & No & 1x closed reduction \\
\hline 5 & 36.7 & 14.2 & 1 & $2 \mathrm{~m}$ & No & $1 \mathrm{x}$ closed reduction \\
\hline 6 & 40.7 & 16.3 & 1 & $4 w$ & No & 1x closed reduction \\
\hline 7 & 39.8 & 4.2 & 2 & $2 w ; 4 w$ & Yes & $2 x$ closed reduction \\
\hline 8 & 35.1 & 18.7 & 1 & $2 \mathrm{~m}$ & No & $1 \mathrm{x}$ closed reduction \\
\hline 9 & 39.6 & 11.0 & 1 & $4 w$ & No & 1x closed reduction \\
\hline
\end{tabular}

to alterations in the APP. They utilized a mathematical algorithm that found that every $1^{\circ}$ of pelvic tilt led to functional correction of radiographic acetabular anteversion of $0.7^{\circ}$. They suggested that surgeons using intraoperative navigation should account for pelvic tilt to better position the acetabular component.

The sole use of anatomic landmarks can lead to acetabular component malpositioning due to variations in pelvic orientation. These variations are largely influenced by sagittal plane balance. ${ }^{21}$ A major advantage of DAA THA is that it facilitates the use of intraoperative fluoroscopy due to the supine position of the patient. Because the patient is supine, there is less alteration of pelvic orientation during surgery. The standing pelvis AP radiograph is a simple way of capturing the patient-specific variations in sagittal spine balance and pelvic tilt. We believe that intraoperatively recreating the standing preoperative AP pelvis view provides a better framework for acetabular component placement. ${ }^{16,22,23,29}$ This is highlighted when evaluating component orientation in patients with extreme pelvic sagittal imbalance (e.g., ankylosing spondylitis), which leads to unintended cup malposition and increased risk of dislocation. 21,30

Accurate interpretation and utilization of intraoperative fluoroscopy with regard to cup placement have an associated learning curve, in addition to the technical learning curve associated with the surgical approach. ${ }^{6,31}$ After this learning period, we believe our method allows the surgeon to gauge accuracy of cup placement with increased validity, as final cup position is measured with similar pelvic orientation as when cup was placed. Studies have shown that DAATHA with fluoroscopy improves accuracy and reduces variability of cup placement compared with freehand DAA THA, ${ }^{32}$ freehand posterior approach THA, ${ }^{6}$ and fluoroscopy-assisted posterior approach THA. $^{23}$ Fluoroscopy has also been shown to improve cup positioning in posterior approach THA compared with freehand methods as well. ${ }^{33}$ Some suggest that surgical approach itself contributes to stability and that optimal safe zones may be approach dependent. ${ }^{23,34}$

Cup abduction angle $<45^{\circ}$ has been associated with decreased rates of polyethylene wear and osteolysis. ${ }^{35,36}$
Although abduction angle is less influenced by pelvic tilt than is anteversion, both can be significantly affected by positional changes; some suggest close monitoring of polyethylene wear in THA patients with severe posterior tilt, though this may be less of an issue with highly cross-linked polyethylene. ${ }^{37}$ Another recent study found that patientspecific geometry had a greater impact of polyethylene wear than head size in THA. ${ }^{38}$ In recent studies regarding native hip impingement, the standing AP pelvis was theorized to better reflect sagittal balance and potentially serve as a better guide for surgical planning and treatment. ${ }^{18,20}$ For these reasons, using the standing functional plane to account for pelvic tilt could minimize cup-related impingement and wear in addition to providing optimal coverage.

Lastly, given that most dislocations occurred within the safe zone, it reaffirms our knowledge that stability after THA is multifactorial. It is possible that other factors that we did not control for, such as femoral offset, femoral version, and patient compliance, led to these dislocations. It could also be that a more advanced metric is needed to identify certain patient subgroups that might require cup placement outside the conventional safe zone. One group suggests that there may not be a distinct safe zone at all, ${ }^{11}$ at least with the posterior approach, or that a spherical safe zone is ideal. ${ }^{34}$ Similar to our findings, most of their dislocations occurred with cups inside the safe zone. Since fluoroscopy is readily available, has been consistently shown to improve accuracy and precision of cup placement, and is most effective with the DAA, continued investigation into ideal cup positioning is warranted in this setting.

\section{Limitations}

One limitation for this study is the use of manual calculation to determine the position of the acetabular component on postoperative AP pelvis radiographs. While this method has been proven to be reproducible, there exist more accurate albeit expensive methods to measure acetabular component positioning such as computed tomography. We suggest that the method provided in this study is precise, reproducible, and economical as demonstrated in the results. A second 
limitation to this study deals with the quality assurance of the measured radiographs. This was best addressed by utilizing the same preoperative technique to recreate the standing AP pelvic film in a supine position; however, no simultaneous quality measurement of variability was performed. A recent study evaluating cup position in DAA THA using methodology similar to ours, also referencing the standing functional plane, found that $95 \%$ of the time the fluoroscopic inclination measurement was between $3.5^{\circ}$ less and $2^{\circ}$ more compared with the postoperative standing AP Xray; similarly, anteversion measurements were between $3.8^{\circ}$ less and $2.2^{\circ}$ more. ${ }^{23}$ We also did not account for other factors known to affect stability that include but are not limited to femoral offset, femoral anteversion, cup size, femoral head size, patient compliance, limb length discrepancy, and soft tissue tension. There was also no control group to reference dislocation rates from this and other approaches. Further analysis of our data to perhaps find a unique safe zone was not performed. Additionally, we acknowledge that there is increased exposure to radiation from fluoroscopy.

\section{Conclusion}

Due to the variety of study designs, surgical approaches and techniques, inconsistencies in defining cup orientation reference frames, measurement error, and patient-specific differences, it is difficult to draw broad conclusions regarding a definitive target zone for cup positioning in THA. Stability after THA is clearly multifactorial, so the ideal safe zone for each patient may vary depending on these factors. An ideal safe zone that completely eliminates the risk of dislocation may not exist. However, redefining the safe zone based upon functional pelvic tilt may further reduce this risk and our results support this. We recommend that future studies investigating acetabular cup positioning control for additional factors mentioned previously and assess different surgical approaches separately.

\section{References}

1 Barrack RL, Krempec JA, Clohisy JC, et al. Accuracy of acetabular component position in hip arthroplasty. J Bone Joint Surg Am 2013;95(19):1760-1768

2 Scheerlinck T. Cup positioning in total hip arthroplasty. Acta Orthop Belg 2014;80(03):336-347

3 Paterno SA, Lachiewicz PF, Kelley SS. The influence of patientrelated factors and the position of the acetabular component on the rate of dislocation after total hip replacement. J Bone Joint Surg Am 1997;79(08):1202-1210

4 Higa M, Tanino H, Abo M, Kakunai S, Banks SA. Effect of acetabular component anteversion on dislocation mechanisms in total hip arthroplasty. J Biomech 2011;44(09):1810-1813

5 Kwon MS, Kuskowski M, Mulhall KJ, Macaulay W, Brown TE, Saleh KJ. Does surgical approach affect total hip arthroplasty dislocation rates? Clin Orthop Relat Res 2006;447(447):34-38

6 Rathod PA, Bhalla S, Deshmukh AJ, Rodriguez JA. Does fluoroscopy with anterior hip arthroplasty decrease acetabular cup variability compared with a nonguided posterior approach? Clin Orthop Relat Res 2014;472(06):1877-1885

7 Callanan MC, Jarrett B, Bragdon CR, et al. The John Charnley Award: risk factors for cup malpositioning: quality improvement through a joint registry at a tertiary hospital. Clin Orthop Relat Res 2011;469(02):319-329

8 Yoon Y-S, Hodgson AJ, Tonetti J, Masri BA, Duncan CP. Resolving inconsistencies in defining the target orientation for the acetabular cup angles in total hip arthroplasty. Clin Biomech (Bristol, Avon) 2008;23(03):253-259

9 Lewinnek GE, Lewis JL, Tarr R, Compere CL, Zimmerman JR. Dislocations after total hip-replacement arthroplasties. J Bone Joint Surg Am 1978;60(02):217-220

10 Timperley AJ, Biau D, Chew D, Whitehouse SL. Dislocation after total hip replacement - there is no such thing as a safe zone for socket placement with the posterior approach. Hip Int 2016;26 (02):121-127

11 Abdel MP, von Roth P, Jennings MT, Hanssen AD, Pagnano MW. What safe zone? The vast majority of dislocated THAs are within the Lewinnek safe zone for acetabular component position. Clin Orthop Relat Res 2016;474(02):386-391

12 Seagrave KG, Troelsen A, Malchau H, Husted H, Gromov K. Acetabular cup position and risk of dislocation in primary total hip arthroplasty. Acta Orthop 2017;88(01):10-17

$13 \mathrm{Ng}$ VY, McShane MA. Understanding acetabular cup orientation: the importance of convention and defining the safe zone. Hip Int 2011;21(06):646-652

14 Murray DW. The definition and measurement of acetabular orientation. J Bone Joint Surg Br 1993;75(02):228-232

15 Philippot R, Wegrzyn J, Farizon F, Fessy MH. Pelvic balance in sagittal and Lewinnek reference planes in the standing, supine and sitting positions. Orthop Traumatol Surg Res 2009;95(01): 70-76

16 Babisch JW, Layher F, Amiot L-P. The rationale for tilt-adjusted acetabular cup navigation. J Bone Joint Surg Am 2008;90(02): 357-365

17 Lembeck B, Mueller O, Reize P, Wuelker N. Pelvic tilt makes acetabular cup navigation inaccurate. Acta Orthop 2005;76(04): 517-523

18 Ross JR, Tannenbaum EP, Nepple JJ, Kelly BT, Larson CM, Bedi A Functional acetabular orientation varies between supine and standing radiographs: implications for treatment of femoroacetabular impingement. Clin Orthop Relat Res 2015;473(04): 1267-1273

19 Eilander W, Harris SJ, Henkus HE, Cobb JP, Hogervorst T. Functional acetabular component position with supine total hip replacement. Bone Joint J 2013;95-B(10):1326-1331

20 Pullen WM, Henebry A, Gaskill T. Variability of acetabular coverage between supine and weightbearing pelvic radiographs. Am J Sports Med 2014;42(11):2643-2648

21 Phan D, Bederman SS, Schwarzkopf R. The influence of sagittal spinal deformity on anteversion of the acetabular component in total hip arthroplasty. Bone Joint J 2015;97-B(08): 1017-1023

22 Slotkin EM, Patel PD, Suarez JC. Accuracy of fluoroscopic guided acetabular component positioning during direct anterior total hip arthroplasty. J Arthroplasty 2015;30(9, Suppl): 102-106

23 Ji W, Stewart N. Fluoroscopy assessment during anterior minimally invasive hip replacement is more accurate than with the posterior approach. Int Orthop 2016;40(01):21-27

24 Maratt JD, Gagnier JJ, Butler PD, Hallstrom BR, Urquhart AG, Roberts KC. No difference in dislocation seen in anterior vs posterior approach total hip arthroplasty. J Arthroplasty 2016; 31(9, Suppl):127-130

25 Matta JM, Shahrdar C, Ferguson T. Single-incision anterior approach for total hip arthroplasty on an orthopaedic table. Clin Orthop Relat Res 2005;441(441):115-124

26 Tripuraneni KR, Munson NR, Archibeck MJ, Carothers JT. Acetabular abduction and dislocations in direct anterior vs posterior total hip arthroplasty: a retrospective, matched cohort study. J Arthroplasty 2016;31(10):2299-2302 
27 Sierra RJ, Raposo JM, Trousdale RT, Cabanela ME. Dislocation of primary THA done through a posterolateral approach in the elderly. Clin Orthop Relat Res 2005;441(441):262-267

28 Masonis JL, Bourne RB. Surgical approach, abductor function, and total hip arthroplasty dislocation. Clin Orthop Relat Res 2002; (405):46-53

29 Tannast M, Langlotz F, Kubiak-Langer M, Langlotz U, Siebenrock KA. Accuracy and potential pitfalls of fluoroscopy-guided acetabular cup placement. Comput Aided Surg 2005;10(5-6):329-336

30 Bedard NA, Martin CT, Slaven SE, Pugely AJ, Mendoza-Lattes SA, Callaghan JJ. Abnormally high dislocation rates of total hip arthroplasty after spinal deformity surgery. J Arthroplasty 2016;31(12):2884-2885

31 Masonis J, Thompson C, Odum S. Safe and accurate: learning the direct anterior total hip arthroplasty. Orthopedics 2008; 31(12, Suppl 2). orthosupersite.com/view.asp?rID $=37187$

32 Jennings JD, Iorio J, Kleiner MT, Gaughan JP, Star AM. Intraoperative fluoroscopy improves component position during anterior hip arthroplasty. Orthopedics 2015;38(11):e970-e975
33 Beamer BS, Morgan JH, Barr C, Weaver MJ, Vrahas MS. Does fluoroscopy improve acetabular component placement in total hip arthroplasty? Clin Orthop Relat Res 2014;472(12):3953-3962

34 Danoff JR, Bobman JT, Cunn G, et al. Redefining the acetabular component safe zone for posterior approach total hip arthroplasty. J Arthroplasty 2016;31(02):506-511

35 Kennedy JG, Rogers WB, Soffe KE, Sullivan RJ, Griffen DG, Sheehan LJ. Effect of acetabular component orientation on recurrent dislocation, pelvic osteolysis, polyethylene wear, and component migration. J Arthroplasty 1998;13(05):530-534

36 Little NJ, Busch CA, Gallagher JA, Rorabeck CH, Bourne RB. Acetabular polyethylene wear and acetabular inclination and femoral offset. Clin Orthop Relat Res 2009;467(11):2895-2900

37 Tezuka $\mathrm{T}$, Inaba $\mathrm{Y}$, Kobayashi $\mathrm{N}$, et al. Influence of pelvic tilt on polyethylene wear after total hip arthroplasty. BioMed Res Int 2015;2015:327217

38 Daniel M, Rijavec B, Dolinar D, Pokorný D, Iglič A, Kralj-Iglič V. Patient-specific hip geometry has greater effect on THA wear than femoral head size. J Biomech 2016;49(16):3996-4001 